\title{
Kinematic diffraction is insufficient to distinguish order from disorder
}

\author{
Michael Baake* \\ Fakultät für Mathematik, Universität Bielefeld, Postfach 100131, 33501 Bielefeld, Germany \\ Uwe Grimm ${ }^{\dagger}$ \\ Department of Mathematics and Statistics, The Open University, Walton Hall, Milton Keynes MK7 6AA, United Kingdom
}

(Received 29 October 2008; published 15 January 2009)

\begin{abstract}
Diffraction methods are at the heart of structure determination of solids. While Bragg-type scattering (pure point diffraction) is a characteristic feature of crystals and quasicrystals, it is not straightforward to interpret continuous diffraction intensities, which are generally linked to the presence of disorder. However, based on simple model systems, we demonstrate that it may be impossible to draw conclusions on the degree of order in the system from its diffraction image. In particular, we construct a family of one-dimensional binary systems which cover the entire entropy range but still share the same purely diffuse diffraction spectrum.
\end{abstract}

DOI: 10.1103/PhysRevB.79.020203

\section{INTRODUCTION}

The inverse problem of reconstructing a structure from its diffraction pattern is one of the most important challenges in materials science. Its degree of complexity increases if one goes beyond simple periodic systems to cover quasicrystals, modulated structures, or complex alloys. In particular, it has been realized that the Bragg diffraction alone is generally insufficient for a complete reconstruction. ${ }^{1-3}$

Currently, an increasing effort is being made to understand and utilize the continuous part of the diffraction pattern; see Refs. 1 and 2 for background and Refs. 4 and 5 for recent applications. However, even in the idealized situation of a perfect diffraction experiment with unlimited resolution, the reconstruction is generally not unique. Already in 1944, Patterson ${ }^{6}$ discussed homometric point sets, which are point sets whose kinematic diffraction patterns coincide, and provided explicit examples to illustrate the ambiguity. It was demonstrated $^{3}$ that it may be possible to lift the ambiguity, and thus to determine the structure uniquely, if higher-order correlations are known. While one can argue that, for structures originating from systems with pure pair-potential interaction (or allowing a description by effective pair potentials; compare Ref. 7), higher-order correlations are determined by the pair correlations, ${ }^{8}$ this is not generally the case, and in practice measurements of higher-order correlations are extremely difficult. The role of phase information in stochastic systems was investigated in Ref. 9.

Here, we want to go one step further, and compare the diffraction patterns of various point sets, ranging from deterministic to fully stochastic, in a parametrized way. We characterize the degree of order by the corresponding (metric) entropy. As we will see below, it is possible to construct families of point sets which span an entire entropy range but share the same kinematic diffraction pattern-proving that diffraction is insensitive even to the degree of order in this case.

We start by giving a brief introduction to some basic notions of mathematical diffraction theory, for a onedimensional (but relevant) setting with scatterers placed on integer positions. Although this is a highly idealized situation that ignores displacement effects, its practical relevance is well known; see Ref. 1 and references therein. Then, we
PACS number(s): 61.05.cc, 02.50.-r, 61.43.-j, 61.44. $-\mathrm{n}$

discuss the diffraction of two binary systems (characterized by two scattering strengths) - a perfectly ordered structure based on a specific deterministic sequence, and a completely random structure based on a coin-tossing experiment. It was observed earlier ${ }^{10}$ that these rather different systems share the same diffraction, and are thus homometric. Finally, we introduce a "Bernoullization" procedure to couple cointossing disorder to a perfectly ordered structure, thus producing partially ordered systems of varying entropy. We employ this procedure to explicitly construct a family of binary systems which are homometric and cover the entire available entropy range from the perfectly ordered (entropy 0 ) to the fully stochastic situation [entropy $\ln (2)]$. Although these systems may not occur naturally, they can be made synthetically. In the simplest scenario, a binary structure can be produced by sequential deposition of layers consisting of two different materials, which makes it possible to realize any desired sequence; see Ref. 11 for an example. More complicated structures are also feasible (see, for instance, Ref. 12) and such artificial materials with designed physical properties will become increasingly important.

\section{DIFFRACTION OF DIRAC COMBS}

To keep arguments simple, we consider the diffraction of one-dimensional systems with pointlike scatterers located at integer points $n \in \mathbb{Z}$. The scattering strengths are given by weights $w_{n}$ for $n \in \mathbb{Z}$, which we assume to be real for simplicity (the setting can be extended to complex weights). The corresponding scattering density is modeled by the Dirac comb

$$
\omega=\sum_{n \in \mathbb{Z}} w_{n} \delta_{n},
$$

where $\delta_{x}$ denotes the normalized point measure (Dirac $\delta$ ) on the real line, located at position $x$. Clearly, all distances between scatterers are integer valued. This implies that the $a u$ tocorrelation (or Patterson) measure $\gamma$, assuming its existence for the moment, is again a Dirac comb on $\mathbb{Z}$,

$$
\gamma=\sum_{m \in \mathbb{Z}} \eta(m) \delta_{m}
$$

with the coefficients $\eta(m)$ obtained as the limits 


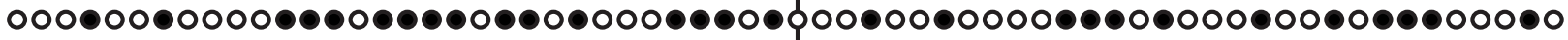

FIG. 1. Central part of the Rudin-Shapiro chain, with open (full) circles representing scattering strengths $w(n)=1[w(n)=-1]$, respectively. The location of the origin $(n=0)$ is indicated by the vertical line.

$$
\eta(m)=\lim _{N \rightarrow \infty} \frac{1}{2 N+1} \sum_{n=-N}^{N} w_{n} w_{n+m} .
$$

The scattering intensity $I(k)$ for wave numbers $k \in \mathbb{R}$ is then determined by the diffraction measure $\hat{\gamma}$, the Fourier transform of the autocorrelation $\gamma$; compare Ref. 13 for background. There are several slightly different versions of the Fourier transform. We prefer to use

$$
\hat{\phi}(k)=\int_{\mathbb{R}} e^{-2 \pi i k x} \phi(x) d x
$$

for a Schwartz function $\phi$, and its standard extension to tempered distributions and measures; see Ref. 14 for details.

For the case of a one-dimensional crystal with $w_{n}=w$ for all $n \in \mathbb{Z}$, we have $\eta(m)=w^{2}$ for all $m \in \mathbb{Z}$; hence $\gamma=w^{2} \delta_{\mathbb{Z}}$, where we use $\delta_{Z}$ as shorthand for the sum $\Sigma_{n \in Z} \delta_{n}$. Its Fourier transform is obtained by Poisson's summation formula, ${ }^{14}$ $\hat{\delta}_{\mathrm{Z}}=\delta_{\mathrm{Z}}$, which gives $\hat{\gamma}=w^{2} \delta_{\mathrm{Z}}$. The diffraction image thus consists entirely of Bragg peaks, located at integer positions $k$, with equal diffraction intensities $I(k)=w^{2}$. The diffraction spectrum in this case is pure point, meaning that it consists of Bragg peaks only. In general, the diffraction measure may comprise three different contributions, $\hat{\gamma}=\hat{\gamma}_{\mathrm{pp}}+\hat{\gamma}_{\mathrm{sc}}+\hat{\gamma}_{\mathrm{ac}}$, where $\hat{\gamma}_{\mathrm{pp}}$ is the pure point part, consisting of a countable sum of $\delta$ peaks. The term $\hat{\gamma}_{\mathrm{ac}}$ corresponds to the absolutely continuous component, which can be described by a locally integrable (and often continuous) non-negative function $I_{\mathrm{ac}}(k)$ of the wave vector $k$. The remainder, if there is any, is called the singular continuous component $\hat{\gamma}_{\mathrm{sc}}$. While it vanishes on the complement of a set $S$ of measure 0 , even within $S$ it never gives weight to any single point. When such a component is present, $S$ can thus not be a countable set. Apart from trivial examples of a diffraction measure that is concentrated on a line in the plane, or similarly on a manifold of lower dimension, typical examples for this strange contribution are diffraction intensities which are supported on a Cantor set or a dense set. A well-known example for the latter phenomenon is the Thue-Morse chain. For an appropriate choice of its scattering strengths, it has a purely singular continuous diffraction measure; see Refs. 15 and 16 for derivations and Refs. 11 and 12 for applications.

If the Dirac comb on $\mathbb{Z}$ is periodic, which means that there is an integer $p>0$ such that $w_{n+p}=w_{n}$ for all $n \in \mathbb{Z}$, the diffraction measure is pure point and supported on the lattice $\mathbb{Z} / p$. It is again periodic, at least with period 1 , but not necessarily with any smaller period. As an example, consider the alternating Dirac comb with $w_{n}=(-1)^{n}$. In this case, $\eta(m)$ $=(-1)^{m}$ for $m \in \mathbb{Z}$, so the autocorrelation is $\gamma=\delta_{2 Z}-\delta_{2 Z+1}$. By Poisson's summation formula and elementary properties of the Fourier transform (such as the behavior under scaling and the convolution theorem), we have

$$
\widehat{\delta_{2 \mathrm{Z}+1}}=[\cos (2 \pi k) / 2] \delta_{\mathrm{Z} / 2},
$$

which leads to the diffraction measure

$$
\hat{\gamma}=[1-\cos (2 \pi k) / 2] \delta_{\mathrm{Z} / 2}=\delta_{\mathrm{Z}+1 / 2} .
$$

In this case, the diffraction spectrum is again pure point, and consists of Bragg peaks of unit intensity at positions $n$ $+1 / 2$, hence on a subset of $\mathbb{Z} / 2$. The fundamental period of $\hat{\gamma}$ is nevertheless still 1. To obtain absolutely or singular continuous components, in line with the classification of Ref. 17 , we thus have to go beyond the periodic situation.

\section{RUDIN-SHAPIRO VERSUS BERNOULLI}

Let us start with a deterministic system without periodicity, based on the well-known binary Rudin-Shapiro chain. We consider the corresponding Dirac comb

$$
\omega_{\mathrm{RS}}=\sum_{n \in Z} w(n) \delta_{n},
$$

where $w: \mathbb{Z} \rightarrow\{ \pm 1\}$ is defined by the recursion

$$
w(4 n+\ell)= \begin{cases}w(n) & \text { for } \ell \in\{0,1\}, \\ (-1)^{n+\ell} w(n) & \text { for } \ell \in\{2,3\},\end{cases}
$$

together with the two initial conditions $w(0)=1$ and $w(-1)$ $=-1$. The resulting system is an aperiodic sequence in 1 and -1 , both appearing equally frequent; see Fig. 1 for a graphical representation. It has many nice properties, such as strict ergodicity and linear patch counting complexity; see Ref. 18 and references therein for details. In particular, these properties imply that this sequence has topological (and metric) entropy 0 .

Since $\omega_{\mathrm{RS}}$ is a Dirac comb on $\mathbb{Z}$, the autocorrelation is of form (1), with coefficients

$$
\eta_{\mathrm{RS}}(m)=\lim _{N \rightarrow \infty} \frac{1}{2 N+1} \sum_{n=-N}^{N} w(n) w(n+m) .
$$

It follows from the unique ergodicity of the RS sequence that all these coefficients (and hence $\gamma$ ) exist, by an application of the ergodic theorem. They are given by $\eta_{\mathrm{RS}}(m)=\delta_{m, 0}$, which is astonishing. The construction of a deterministic sequence with vanishing two-point correlations was the original (and independent) motivation of Rudin and Shapiro, thus answering a question in the theory of Fourier series.

Let us prove this property by a simple, explicit argument. Consider $a_{m}=\eta_{\mathrm{RS}}(m)$ together with

$$
b_{m}=\lim _{N \rightarrow \infty} \frac{1}{2 N+1} \sum_{n=-N}^{N}(-1)^{n} w(n) w(n+m),
$$

which also exist (by another application of the ergodic theorem). Clearly, one has $a_{0}=1$ and $b_{0}=0$ because $w(n)^{2}=1$ for all $n \in \mathbb{Z}$. Then, considering $m \bmod 4$ and splitting the sums in the definition of $a_{m}$ and $b_{m}$ accordingly, recursion relation (2) implies

$$
a_{4 m}=\left[1+(-1)^{m} / 2\right] a_{m}, \quad a_{4 m+2}=0,
$$




$$
\begin{aligned}
& a_{4 m+1}=\frac{1-(-1)^{m}}{4} a_{m}+\frac{(-1)^{m}}{4} b_{m}-\frac{1}{4} b_{m+1}, \\
& a_{4 m+3}=\frac{1-(-1)^{m}}{4} a_{m+1}-\frac{(-1)^{m}}{4} b_{m}+\frac{1}{4} b_{m+1} .
\end{aligned}
$$

Similarly, one finds

$$
\begin{gathered}
b_{4 m}=0, \quad b_{4 m+2}=\frac{(-1)^{m}}{2}\left(b_{m}+b_{m+1}\right), \\
b_{4 m+1}=\frac{1-(-1)^{m}}{4} a_{m}-\frac{(-1)^{m}}{4} b_{m}+\frac{1}{4} b_{m+1}, \\
b_{4 m+3}=-\frac{1+(-1)^{m}}{4} a_{m+1}-\frac{(-1)^{m}}{4} b_{m}+\frac{1}{4} b_{m+1} .
\end{gathered}
$$

Using the initial data, these recursion relations imply that $a_{m}=b_{m}=0$ for all integers $m \neq 0$. This result shows that the autocorrelation and diffraction measures of the binary RudinShapiro Dirac comb $\omega_{\mathrm{RS}}$ are simply

$$
\gamma_{\mathrm{RS}}=\delta_{0} \text { and } \widehat{\gamma_{\mathrm{RS}}}=\lambda,
$$

where $\lambda$ denotes Lebesgue measure. In other words, the diffraction measure is purely absolutely continuous, and consists of a constant background (of height 1) only. The extinction of all Bragg peaks is due to the balanced choice of weights. This is convenient for the theoretical argument, but also relevant in practice ${ }^{19}$ when disregarding thermal displacement. Note that all arguments can be extended to mixed spectra.

Perhaps the most elementary stochastic system is based on the classic coin-tossing (or Bernoulli) experiment. We consider a stochastic Dirac $\operatorname{comb}^{20}$ on $\mathbb{Z}$,

$$
\omega_{B}=\sum_{n \in Z} W_{n} \delta_{n},
$$

where $\left(W_{n}\right)_{n \in Z}$ is a family of independent identically distributed (iid) random variables, with probabilities $\mathrm{P}\left(W_{n}=1\right)=p$ and $\mathrm{P}\left(W_{n}=-1\right)=1-p$, where $0 \leq p \leq 1$. The corresponding (metric) entropy is

$$
H(p)=-p \ln (p)-(1-p) \ln (1-p),
$$

which satisfies $0 \leq H(p) \leq \ln (2)$. It attains the extremal values for $p=0$ and $p=1$, where $H=0$ is minimal, and for $p$ $=\frac{1}{2}$, where $H=\ln (2)$ is maximal.

The autocorrelation $\gamma_{B}=\sum_{n \in Z} \eta_{B}(m) \delta_{m}$ is once again a pure point measure that is supported on $\mathbb{Z}$, with autocorrelation coefficients given by

$$
\eta_{B}(m)=\lim _{N \rightarrow \infty} \frac{1}{2 N+1} \sum_{n=-N}^{N} W_{n} W_{n+m}
$$

for $m \in \mathbb{Z}$. These coefficients almost surely exist, by an application of the strong law of large numbers (see below), for all $m \in \mathbb{Z}$, and satisfy

$$
\eta_{B}(m)= \begin{cases}1, & m=0, \\ (2 p-1)^{2}, & m \neq 0 .\end{cases}
$$

This statement can be proved as follows. It obviously holds for $m=0$, so consider some fixed $m \neq 0$. The products $Z_{n}$ $:=W_{n} W_{n+m}$ form a family $\left(Z_{n}\right)_{n \in \mathrm{Z}}$ of identically distributed random variables, which take values 1 and -1 with probabilities $p^{2}+(1-p)^{2}$ and $2 p(1-p)$, respectively. These new random variables are not independent, but we can split the sum in Eq. (4) into two sums (for instance, according to even and odd values of $\left[\frac{n}{m}\right]$, the largest integer smaller than or equal to $\left.\frac{n}{m}\right)$. The resulting two sums each comprise pairwise independent random variables. An application of the strong law of large numbers in its formulation by Etemadi ${ }^{21}$ then shows that each sum almost surely converges (as $N \rightarrow \infty$ ) to the expectation value of any of the single random variables in the sum, which is $\frac{1}{2}(2 p-1)^{2}$. Hence, the diffraction measure of the stochastic Dirac comb $\omega_{B}$ almost surely is

$$
\widehat{\gamma_{\omega_{B}}}=(2 p-1)^{2} \delta_{\mathrm{Z}}+4 p(1-p) \lambda,
$$

where $\lambda$ again denotes Lebesgue measure. In other words, the diffraction spectrum comprises a constant background of intensity $4 p(1-p)$ for any value of the wave number $k$ and Bragg peaks of intensity $(2 p-1)^{2}$ at integer $k$. Note that for the perfectly ordered cases $p=0$ and $p=1$, the background vanishes, while the Bragg peaks vanish for the maximally disordered case $p=\frac{1}{2}$. At this value of $p$, the diffraction measure coincides with that of the Rudin-Shapiro chain.

This establishes the homometry of the deterministic binary Rudin-Shapiro chain (with entropy 0) and the completely random Bernoulli chain with $p=\frac{1}{2}$ [with entropy $\ln (2)]$, as originally observed in Ref. 10. Coupling the two systems in a suitable way, we shall now extend this to an entire family that covers the intermediate entropy range.

\section{BERNOULLIZATION}

The Bernoulli chain discussed above is an example of a completely random and interaction-free system. In view of real-world examples, it is interesting to explore what happens if one imposes the influence of coin tossing on the order of a deterministic system. This can be realized in many different ways. Here, we focus on binary sequences and modify them by an iid family of Bernoulli variables.

Consider a bi-infinite binary sequence $S \in\{ \pm 1\}^{Z}$ which we assume to be uniquely ergodic. Then, the corresponding Dirac comb $\omega_{S}=\Sigma_{n \in \mathbb{Z}} S_{n} \delta_{n}$ possesses the unique (natural) autocorrelation $\gamma_{S}=\Sigma_{m \in Z} \eta_{S}(m) \delta_{m}$ with the autocorrelation coefficients $\eta_{S}(m)$, where $\eta_{S}(0)=1$ by construction.

Let $\left(W_{n}\right)_{n \in Z}$ be an iid family of random variables where each takes values +1 and -1 with probabilities $p$ and $1-p$. The Bernoullization of $\omega_{S}$ is the random Dirac comb

$$
\omega:=\sum_{n \in \mathbb{Z}} S_{n} W_{n} \delta_{i},
$$

which emerges from $\omega_{S}$ by independently changing the sign of each $S_{n}$ with probability $1-p$. Setting $Z_{n}:=S_{n} W_{n}$ defines a new family of independent (though in general not identically 
distributed) random variables, with values in $\{ \pm 1\}$. Despite this modification, the autocorrelation $\gamma$ of $\omega$ almost surely exists and can be determined via its autocorrelation coefficients $\eta(m)$ as follows. Since one always has $\eta(0)=\eta_{S}(0)$ $=1$, let $m \neq 0$ and consider, for large $N$, the sum

$$
\begin{aligned}
\frac{1}{2 N+1} & \sum_{n=-N}^{N} Z_{n} Z_{n+m} \\
& =\frac{1}{2 N+1}\left(\sum_{(+,+)}+\sum_{(-,-)}-\sum_{(+,-)}-\sum_{(-,+)}\right) W_{n} W_{n+m},
\end{aligned}
$$

which is split according to the value of $\left(S_{n}, S_{n+m}\right)$. Each of the four sums can then be handled in the same way as in the argument for the Bernoulli chain above, thus contributing $(2 p-1)^{2}$ times the frequency of the corresponding sign pair. Observing that the overall signs are the products $S_{n} S_{n+m}$, it is clear that as $N \rightarrow \infty$, one (almost surely) obtains $\eta(m)$ $=(2 p-1)^{2} \eta_{S}(m)$ for all $m \neq 0$. This shows that the new autocorrelation almost surely is $\gamma=(2 p-1)^{2} \gamma_{S}+4 p(1-p) \delta_{0}$ where $\gamma_{S}$ is the unique autocorrelation of $\omega_{S}$.

Let us apply this Bernoullization procedure to the RudinShapiro chain. Denote by $\omega$ the random Dirac comb obtained from the Bernoullization (with parameter $p$ ) of the binary Rudin-Shapiro chain. Then, the autocorrelation measure almost surely exists and reads $\gamma=\delta_{0}$, independently of $p$. This means that the random Dirac combs $\omega$, even for different values of $p$, are almost surely homometric, and share the purely absolutely continuous diffraction measure $\hat{\gamma}=\lambda$.

Note that this example explores the full entropy range of Eq. (3): the Bernoulli case (with $p=\frac{1}{2}$ ) has entropy $\ln (2)$, the maximal value for a binary system, while Rudin-Shapiro has entropy 0 , and the parameter $p$ interpolates continuously between the two limiting cases. The solution of the corresponding inverse problem is thus highly degenerate. Unless additional information is available, for instance, via higher-order correlations, one possible strategy could employ a maximum entropy method, ${ }^{22}$ singling out the Bernoulli comb.
Both the Bernoullization procedure and the specific onedimensional examples immediately generalize to higher dimensions by taking direct product structures. In particular, the product of $d$ Rudin-Shapiro chains results in a deterministic system in $d$ space with the same purely absolutely continuous diffraction measure as the corresponding cointossing model. Consequently, our above conclusions extend to this case. This means that one can also produce examples with lower-rank entropy, which is a phenomenon that occurs in dimensions $d \geq 2$.

\section{CONCLUDING REMARKS}

Diffraction methods provide the most important approach to structure determination. The presence of Bragg diffraction clearly indicates an ordered structure, though the discovery of quasicrystals ${ }^{23}$ in the 1980 s showed that pure point diffraction occurs in more general systems than just conventional crystals. To date, the precise atomic structure of quasicrystalline alloys is still not completely understood; there is evidence that entropy plays an important role in stabilizing quasicrystalline structures, and that some disorder may be an inherent feature of these alloys. Like for many ordinary crystals, diffuse scattering is present in experimental diffraction patterns of even the best-known quasicrystals, and there is an increasing effort to explore the information contained in the diffuse diffraction intensity ${ }^{1,2}$; see Ref. for a recent example. It is tempting to draw conclusions about the degree of order in a structure on the basis of the observed diffuse scattering intensity. However, as our explicit example demonstrates, such conclusions have to be considered carefully since the relation between diffuse scattering and disorder is far from simple.

This work was supported by the German Research Foundation (DFG), within the CRC 701, and by EPSRC-GB via Grant No. EP/D058465. It is a pleasure to thank the School of Mathematics and Physics at the University of Tasmania for their kind hospitality.

\footnotetext{
*mbaake@math.uni-bielefeld.de

†u.g.grimm@open.ac.uk

${ }^{1}$ T. R. Welberry, Diffuse X-ray Scattering and Models of Disorder (Oxford University Press, Oxford, 2004)

${ }^{2}$ R. B. Neder and T. Proffen, Diffuse Scattering and Defect Structure Simulations (Oxford University Press, Oxford, 2008).

${ }^{3}$ F. A. Grünbaum and C. C. Moore, Acta Crystallogr., Sect. A: Found. Crystallogr. 51, 310 (1995).

${ }^{4}$ M. de Boissieu et al., Phys. Rev. Lett. 95, 105503 (2005).

${ }^{5}$ M. Kopecký et al., Phys. Rev. Lett. 100, 195504 (2008).

${ }^{6}$ A. L. Patterson, Phys. Rev. 65, 195 (1944).

${ }^{7}$ W. Schweika and A. E. Carlsson, Phys. Rev. B 40, 4990 (1989).

${ }^{8}$ D. M. C. Nicholson et al., J. Phys.: Condens. Matter 18, 11585 (2006).

${ }^{9}$ T. R. Welberry and R. L. Withers, J. Appl. Crystallogr. 24, 18 (1991).

${ }^{10}$ M. Höffe and M. Baake, Z. Kristallogr. 215, 441 (2000).

${ }^{11}$ F. Axel and H. Terauchi, Phys. Rev. Lett. 66, 2223 (1991).

${ }^{12}$ W. Steurer and D. Sutter-Widmer, J. Phys. D 40, R229 (2007).
}

${ }^{13}$ A. Hof, Commun. Math. Phys. 169, 25 (1995).

${ }^{14}$ L. Schwartz, Théorie des Distributions, 3rd ed. (Herman, Paris, 1998).

${ }^{15}$ S. Kakutani, in Proceedings of the Sixth Berkeley Symposium on Mathematical Statistics and Probability, edited by L. M. Le Cam, J. Neyman, and E. L. Scott (University of California Press, Berkeley, 1972), pp. 319-326.

${ }^{16}$ M. Baake and U. Grimm, J. Phys. A: Math. Theor. 41, 422001 (2008).

${ }^{17}$ M. Baake and R. V. Moody, J. Reine Angew. Math. 573, 61 (2004).

${ }^{18}$ M. Queffélec, Substitution Dynamical Systems-Spectral Analysis (Springer, Berlin, 1987).

${ }^{19}$ S. Dietrich and W. Fenzl, Phys. Rev. B 39, 8900 (1989).

${ }^{20}$ M. Baake and R. V. Moody, J. Phys. A 31, 9023 (1998).

${ }^{21}$ N. Etemadi, Z. Wahrscheinlichkeitstheor. Verwandte Geb. 55, 119 (1981).

${ }^{22}$ S. Guiaşu and A. Schenitzer, Math. Intell. 7, 42 (1985).

${ }^{23}$ D. Shechtman et al., Phys. Rev. Lett. 53, 1951 (1984). 